\title{
Diacronie
}

Studi di Storia Contemporanea

$N^{\circ} 15,3 \mid 2013$

Spazi, percorsi e memorie

\section{Michela Ponzani, Guerra alle donne. Partigiane, vittime di stupro, «amanti del nemico»}

\section{Andrea Martini}

\section{(2) OpenEdition}

Journals

\section{Edizione digitale}

URL: http://journals.openedition.org/diacronie/628

DOI: 10.4000/diacronie.628

ISSN: 2038-0925

\section{Editore}

Association culturelle Diacronie

Notizia bibliografica digitale

Andrea Martini, « Michela Ponzani, Guerra alle donne. Partigiane, vittime di stupro, «amanti del nemico» », Diacronie [Online], № 15, 3 | 2013, documento 16, online dal 01 octobre 2013, consultato il 10 décembre 2020. URL : http://journals.openedition.org/diacronie/628 ; DOI : https://doi.org/10.4000/ diacronie. 628 


\section{Diacronie}

\section{RECENSIONE:}

\section{Michela PONZANI, Guerra alle donne. Partigiane, vittime di stupro, «amanti del nemico», Torino, Einaudi, 2012, 313 pp.}

a cura di Andrea MARTINI*

Guerra alle donne di Michela Ponzani ${ }^{1}$ vorrebbe offrire una prospettiva nuova da cui osservare la Seconda guerra mondiale e in effetti vi riesce, sollevando numerosi interrogativi e proponendo alcune risposte. L'opera, collocandosi tra gli studi di genere, si ricollega ad una serie di volumi dedicati alla partecipazione delle donne alla guerra. Si ricordi che ad occuparsi per prime della presenza femminile nelle bande partigiane furono Rachele Farina e Anna Maria Bruzzone con l'opera La Resistenza taciuta ${ }^{2}$; altri punti di riferimento sono le ricerche curate da Anna Bravo Donne e uomini nelle guerre mondiali3 e In guerra senz'armit. La prima, partendo dalla sintesi di un convegno dedicato al tema, si preoccupava di fare luce sul ruolo giocato dai civili durante il conflitto. La seconda aveva il merito di introdurre due concetti utilizzati ancor oggi dagli storici: maternage di massa e resistenza civile, quest'ultimo "importato"

\footnotetext{
${ }^{1}$ L'autrice è una ricercatrice romana che si è occupata sino ad oggi della Resistenza e delle stragi nazifasciste, in particolare quella delle Fosse Ardeatine. Tra le sue pubblicazioni si ricordi: CONTI, Davide, PONZANI, Michela, Lo Stato repubblicano e via Rasella, in BENTIVEGNA, Rosario (a cura di), Achtung banditen! Prima e dopo via Rasella, Milano, Mursia, 2004, PONZANI, Michela, L'eredità della Resistenza nell'Italia repubblicana tra retorica celebrativa e contestazione di legittimità (1945-1963), in Annali della Fondazione L. Einaudi, XXXVIII, Leo S. Olschki Editore, 2004, pp. 259-307 e PONZANI, Michela, L'offensiva giudiziaria antipartigiana nell'Italia repubblicana (1945-1960), Roma, Aracne, 2008. Di recente l'autrice ha collaborato alla commissione storica italo tedesca chiamata dai Ministeri degli Esteri dei rispettivi paesi ad indagare i fatti della Seconda guerra mondiale. La relazione finale (2012) è disponibile sul sito all'indirizzo: URL: < http://www.villavigoni.it/index.php?id=76\&L=1 > [consultato il 23 marzo 2013].

2 FARINA, Rachele, BRUZZONE, Anna Maria (a cura di), La Resistenza taciuta. Dodici vite di partigiane piemontesi, Milano, La Pietra, 1976.

3 BRAVO, Anna (a cura di), Donne e uomini nelle guerre mondiali, Roma, Laterza, 1991.

4 BRAVO, Anna, In guerra senz'armi. Storie di donne 1940-1945, Roma, Laterza, 1995.
} 
dall'opera francese di Jacques Semelin Sans armes face à Hitler ${ }^{5}$. Resistenza civile consente di ampliare l'orizzonte della resistenza, fino a quel momento strettamente legato alle formazioni partigiane. Il concetto di maternage di massa illustra come la donna nel corso della contesa abbia esteso il suo lato materno destinandolo a chiunque ne abbisognasse, specie i soldati all'indomani della tragedia dell'8 settembre. Tra la storiografia più recente si possono menzionare due opere. L'opera di Gabriella Gribaudi, Guerra totale 6 rivela un carattere peculiare della guerra, che fece più vittime tra i civili che tra i soldati; la ricerca di Patrizia Gabrielli Scenari di guerra, parole di donne $^{7}$, raccoglie le testimonianze di donne che, da posizioni differenti e con ruoli diversi, presero parte al conflitto e lasciarono per iscritto presso l'archivio diaristico nazionale la loro testimonianza.

L'opera di Ponzani Guerra alle donne si inserisce, sin dal titolo, all'interno della visione, proposta da Gribaudi, di una guerra totale che, come tale, colpì prevalentemente le donne. L'autrice, dunque, pare condividere il concetto espresso da Ernesto Galli della Loggia che ribattezzò quella contesa guerra femminile ${ }^{8}$, ma adoperandolo in senso quasi opposto: non ne fruisce per evidenziare il ruolo di emancipazione svolto dalla guerra, bensì quello di "bersaglio strategico" incarnato dalle donne. Non sono da trascurare nemmeno le categorie adoperate dall'autrice nel sottotitolo: partigiane, vittime di stupro, "amanti del nemico", inquadrate all'interno della medesima opera come forse mai era accaduto.

Il volume, strutturato in dieci capitoli, fa perno su numerose testimonianze ricavate prevalentemente da due fondi. Il Fondo Rai La mia guerra conservato presso l'INSMLI (a Milano) è costituito da trascrizioni di una serie di interviste compiute ai civili attorno al 1990, in occasione di una trasmissione di Rai3 - La mia guerra - che dava voce alle esperienze di guerra dei civili. L'archivio era già stato esplorato da alcuni studiosi, tra cui Anna Bravo, ma mai con la stessa profondità per la quale si distingue Ponzani. L'altro fondo è quello dell'Archivio della Memoria delle donne, custodito presso il Dipartimento di Discipline storiche dell'Università di Bologna, che raccoglie le testimonianze di diverse donne partigiane.

\footnotetext{
5 SEMELIN, Jacques, Sans armes face à Hitler. La Résistance civile en Europe (1939-1943), Paris, Payot, 1989.

${ }^{6}$ GRIBAUDI Gabriella, Guerra totale. Tra bombe alleate e violenze naziste Napoli e il fronte meridionale 1940-1944, Torino, Bollati Boringhieri, 2005.

${ }^{7}$ GABRIELLI Patrizia, Scenari di guerra, parole di donne, Bologna, Il Mulino, 2007.

${ }^{8}$ GALLI DELLA LOGGIA, Ernesto, Una guerra femminile? Ipotesi sul mutamento dellideologia e dellimmaginario occidentali tra il 1939 e il 1945, in BRAVO, Anna (a cura di), Donne e uomini nelle guerre mondiali, Roma, Laterza, 1991, pp. 3-28
} 
L'autrice dedica attenzione anche agli anni che precedono la guerra, allo scopo di rilevare l'origine dell'antifascismo che alcune donne manifestano durante il periodo 1943-1945, ma il cui seme si impianta durante gli anni Trenta e nei primi anni di guerra, in cui si assiste ad un marcato peggioramento delle condizioni di vita. La cultura patriarcale fascista soffoca le italiane; alcune tra loro maturano un sentimento di disagio accresciuto negli ambienti familiari e scolastici. Sono le privazioni, i divieti, le imposizioni che diffondono in molte donne un desiderio di ribellione. Esemplificativa la testimonianza di Maria Teresa Regard, futura gappista, che comincia ad affrancarsi dalla cultura fascista quando viene a sapere che "le donne non avrebbero potuto iscriversi alle facoltà scientifiche" e che non possono "accedere a determinate carriere e lavori”»9. Si tratta quindi di una resistenza privata, che solo in un successivo momento diverrà "politica".

Abbandonare casa e il ruolo tradizionale assegnato non è facile: innanzitutto c'è la paura della morte, vi è la consapevolezza che dedicarsi alla guerriglia equivalga ad imbattersi in diversi pericoli e c'è inoltre la difficoltà di farsi accettare dal gruppo. Anche i partigiani, infatti, sono imbevuti della stessa cultura maschilista dei fascisti e dunque stentano ad accettare l'altro sesso. Le partigiane conducono più lotte, quella esterna contro il nemico, quella contro i partigiani stessi per ottenerne la fiducia, ma anche una guerra interiore.

Dai resoconti di Guerra delle donne risulta poco veritiera la nozione di «resistenza di massa» sostenuta da una parte della memorialistica resistenziale: molte donne e uomini non prendono parte alla lotta antifascista, non se la sentono o non la condividono. Indubbiamente le donne scontano l'ostilità dei partigiani, ma anche la comunità civile si dimostra talvolta maldisposta. Del resto la strategia dei tedeschi vuol fare "piazza pulita" di molte comunità compiendo pesanti rappresaglie, eliminando così l'humus da cui le formazioni partigiane sorgono. Creare un cortocircuito tra popolazione civile e partigiani è la strategia nemica, avvertita debitamente anche dalle donne.

Il capitolo quinto conferma la volontà dell'autrice di evidenziare come le donne diventino dei "bersagli strategici": durante la guerra esse sono le vittime, assieme ad anziani e bambini, dei bombardamenti aerei di entrambi gli schieramenti condotti per fiaccare il morale dei civili. Durante l'attacco aereo le donne si trasformano in scudi umani per proteggere i loro figli.

I capitoli centrali della ricerca tendono a spostare l'attenzione sui soprusi subiti in diversi contesti: dalle sofferenze quotidiane, a quelle patite nei lager, alle torture e agli

9 PONZANI, Michela, Guerra alle donne, Torino, Einaudi, 2012, p. 33. 
stupri sofferti nelle caserme della Repubblica Sociale Italiana ${ }^{10}$. Interessante è l'interpretazione che l'autrice propone delle violenze inflitte dai repubblichini, che paiono accanirsi sulle donne non solo per estorcere informazioni, ma anche per punizione, dal momento che queste hanno abbandonato il ruolo tradizionale assegnato loro:

a emergere con più forza da queste testimonianze è infatti la cultura militaremaschile dei «carnefici», il substrato mentale, il loro retroterra educativo che spinge a punire le donne che hanno osato ribellarsi al regime e che hanno voluto lottare per emancipare se stesse da quel ruolo sociale inferiorizzante e sottomesso di brave mogli ubbidienti e madri sacrificali ${ }^{11}$.

Nelle parole delle testimoni emerge la loro difficoltà a pronunciarsi sull'argomento: il silenzio è usato come riparo per continuare a vivere, nonostante le sofferenze patite, come bene aveva documentato lo storico Tommaso Baris con la ricerca Tra due fuochi $^{12}$. È emblematico il titolo del settimo capitolo - Ricordare l'indicibile - in cui vi è, tra le altre, una testimonianza esemplare ${ }^{13}$ : quella di una donna sfollata tra le colline attorno a Marzabotto, proprio a ridosso della strage del settembre-ottobre 1944. Rimasta sola (il marito è deportato in Germania), la donna viene stuprata ripetutamente dai tedeschi. Le sue parole molto toccanti sono parzialmente riportate:

Mio cognato mi implorava che andassi su perché se no li uccidevano tutti [...] arrivò in casa anche il prete che diceva: «fatti coraggio, fatti coraggio». [...] Poi il prete, 'sto padre continuava che li voleva convincere in tanti modi, che poi non capivano niente, e all'ultimo gli dissero : «padre, vuol morire assieme a tutti gli altri o vuol tornare da dov'è venuto ?». Lui stette lì un po', a pensare e poi... prima di andare via mi disse: «mettiti nelle mani di Dio». Ma io non ero nelle mani di Dio, ero nelle mani... non so neanche come definirli. E poi mi buttarono su come a buttare su una cosa, su per una scala che andava su nella camera. E fui di quella storia lì fino a

\footnotetext{
${ }^{10}$ Importante ricordare come Ponzani ammetta e tratti tanto delle violenze nazifasciste quanto di quelle inflitte dagli alleati. Cfr. Capitolo ottavo «Marocchinate», pp. 223-252.

${ }^{11}$ PONZANI, Michela, op. cit., p. 172.

${ }^{12}$ BARIS, Tommaso, Tra due fuochi. Esperienza e memoria della guerra lungo la Linea Gustav, Roma-Bari, Laterza, 2003.

${ }^{13}$ La testimonianza è riportata come rivela la stessa autrice anche in VENTUROLI, Cinzia, $L a$ violenza taciuta. Percorsi di ricerca sugli abusi sessuali fra il passaggio e l'arrestarsi del fronte, in GAGLIANI, Dianella, et al. (a cura di), Donne Guerra Politica: esperienze e memorie della Resistenza, Bologna, Clueb, 2000, pp. 114-117
} 
sera. Poi andavano e venivano, non so se erano 5 o 6 , quanti erano. [...] Degli schiaffi, degli sputi... preferivo in quel momento lì mi avessero uccisa ${ }^{14}$.

La testimonianza parla da sé, ma necessita di qualche osservazione. Le parole appaiono uno schermo difensivo adoperato in risposta alla tragicità del fatto. Nel corso di una conferenza ${ }^{15}$ l'autrice ha confidato che questa donna rivelò per la prima volta lo stupro solo nel 1994, durante un'intervista, confessando una tragedia a lungo celata persino al marito, che non aveva neppure desiderato ascoltare la moglie e che, ritornato in Patria dopo aver visto lui stesso "l'inferno", aveva stabilito di ricominciare a vivere condannando all'oblio le tragedie di entrambi.

Il silenzio avvolge anche altre donne, ovvero «le amanti del nemico», coloro che ebbero - o furono accusate (spesso a causa delle maldicenze di qualche vicino/a) di avere avuto relazioni d'amore con i tedeschi e i fascisti. Ponzani riconosce come punizione prediletta nei confronti delle collaborazioniste la rasatura dei capelli. È interessante notare come queste donne vengano rappresentate in alcune memorie con «caratteristiche non femminili» ${ }^{16}$. La studiosa si interroga sulle ragioni del silenzio imposto anche a chi forse visse storie d'amore - più o meno importanti - durante quegli anni: evidentemente quelle esperienze fuoriescono dal canone patriottico della donna partigiana.

Il libro termina tratteggiando la situazione del dopoguerra, rivelando come i molti sacrifici compiuti dalle donne siano stati a lungo negati, le loro sofferenze e tragedie stentino ancor oggi ad emergere.

I temi messi in campo dall'autrice sono molteplici, così come le ragioni per leggere questo volume. Un libro che non solo arricchisce le testimonianze in nostro possesso dei civili, veri protagonisti della guerra totale, ma che solleva anche molti interrogativi. Si può parlare di guerra alle donne? Quanto sono distinte e ben delineabili le categorie proposte dall'autrice? Le donne hanno vinto in questa guerra? Domande a cui è difficile trovare risposta. Forse, però, un'analisi più dettagliata sul dopoguerra consentirebbe di risolvere per lo meno l'ultimo quesito. In effetti gli accenni dell'autrice sulla magistratura, che durante il processo di epurazione si dimostrò di mentalità piuttosto conservatrice, induce a pensare che per le donne la vittoria fosse ancora lontana, almeno a livello giuridico, sebbene la conquista del voto politico rappresentasse un

\footnotetext{
14 PONZANI, Michela, op. cit., p. 215.

15Michela Ponzani ha partecipato al progetto didattico "Verso il 25 aprile", realizzato dal Liceo A. Messedaglia (Verona); la conferenza dove l'autrice ha presentato il suo libro si è svolta il 16 marzo 2013.

16PONZANI, Michela, op. cit., p. 258.
} 
traguardo importante. Attorno alla questione, del resto, ruota un dibattito storiografico aperto.

Il merito della ricercatrice è quello di perlustrare temi poco dibattuti, quali lo stupro durante la seconda guerra mondiale e il trattamento delle amanti del nemico: quest'ultime, tra l'altro, stentano ancora ad avere una dignità storica in Italia.

L'autrice auspica di impiegare le testimonianze nel modo più neutro possibile senza farsi eccessivamente trasportare dalla carica emotiva di cui sono imbevute, ma è difficile constatare se abbia o meno rispettato il suo auspicio. Ponzani, in fondo, non rinuncia mai al carattere di denuncia del volume, che non contrasta comunque con il suo valore scientifico. Il risultato è un pregevole compromesso. Non vi è invece un'analisi più prettamente linguistica, che arricchirebbe l'analisi storica di queste preziose testimonianze. Decodificare lo schermo di pudore (come lo definisce l'autrice) utilizzato da molte protagoniste è un compito che richiederebbe una più stretta collaborazione tra storici e linguisti.

Infine, mi preme sottolineare l'attenzione data al collaborazionismo nella sua declinazione femminile. L'autrice mostra come le donne furono protagoniste attive di entrambi i fronti, quello partigiano e quello collaborazionista. L'analisi di Ponzani ha perciò il merito di ampliare il quadro storiografico preesistente sul collaborazionismo. Un fenomeno, quest'ultimo, che stride con la memoria pubblica del "buon italiano", quello da molti storici viene oramai definito come un vero mito ${ }^{17}$. Pensare che molti italiani ed italiane vestirono i panni del collaborazionista non deve più "spaventare", così come non si deve esitare a parlare apertamente della Seconda guerra mondiale in termini di guerra alle donne.

${ }_{17}$ Cfr. DEL BOCA, Angelo, Italiani, brava gente? : un mito duro a morire, Vicenza, Neri Pozza, 2010; FOCARDI, Filippo, Il cattivo tedesco e il bravo italiano: la rimozione delle colpe della seconda guerra mondiale, Roma-Bari, Laterza, 2013. 


\section{* L'autore}

Andrea Martini, ha conseguito la Laurea in storia presso l'Università di Padova nel 2011 con una tesi intitolata La strategia della tensione e il neofascismo radicale. Attualmente è iscritto al corso di Laurea magistrale in scienze storiche dell'Università di Padova; si occupa dell'epurazione delle collaborazioniste fasciste.

URL:< http://www.studistorici.com/progett/autori/\#Martini >

\section{Per citare questo articolo:}

MARTINI, Andrea, «Recensione: Michela PONZANI, Guerra alle donne. Partigiane, vittime di stupro, «amanti del nemico», Torino, Einaudi, 2012, 313 pp.», Diacronie. Studi di Storia Contemporanea : Spazi, percorsi e memorie, 29/10/2013,

URL:<http://www.studistorici.com/2013/10/29/martini_numero_15/ >

\section{Diacronie Studi di Storia Contemporanea 3 www.diacronie.it}

Risorsa digitale indipendente a carattere storiografico. Uscita trimestrale.

\section{redazione.diacronie@hotmail.it}

Comitato di redazione: Marco Abram - Jacopo Bassi - Luca Bufarale - Alessandro Cattunar - Elisa Grandi - Deborah Paci - Fausto Pietrancosta - Matteo Tomasoni - Luca Zuccolo 